\title{
Earlier Versus Later Augmentation with an Antipsychotic Medication in Patients with Major Depressive Disorder Demonstrating Inadequate Efficacy in Response to Antidepressants: A Retrospective Analysis of US Claims Data
}

\author{
Irina Yermilov • Mallik Greene · Eunice Chang • Ann Hartry • \\ Tingjian Yan $\cdot$ Michael S. Broder
}

Received: September 21, 2018 / Published online: November 19, 2018

(C) The Author(s) 2018

\section{ABSTRACT}

Introduction: There is little evidence regarding the most effective timing of augmentation of antidepressants (AD) with antipsychotics (AP) in patients with major depressive disorder (MDD) who inadequately respond to first-line $\mathrm{AD}$ (inadequate responders). The study's objective was to understand the association between timing of augmentation of AD with $\mathrm{AP}$ and overall healthcare costs in inadequate responders.

Methods: Using the Truven Health MarketS$\operatorname{can}^{\circledR}$ Medicaid, Commercial, and Medicare

Enhanced Digital Features To view enhanced digital features for this article go to https://doi.org/10.6084/ m9.figshare.7297541.

Electronic supplementary material The online version of this article (https://doi.org/10.1007/s12325018-0838-2) contains supplementary material, which is available to authorized users.

I. Yermilov · E. Chang · T. Yan · M. S. Broder Partnership for Health Analytic Research (PHAR), LLC, 280 S. Beverly Dr., Beverly Hills, CA 90212, USA

M. Greene $(\bowtie)$

Otsuka Pharmaceutical Development and

Commercialization, Inc., 508 Carnegie Center, Princeton, NJ 08540, USA

e-mail: mallik.greene@otsuka-us.com

A. Hartry

Lundbeck, LLC, 6 Parkway North, Deerfield, IL 60015, USA
Supplemental databases (7/1/09-12/31/16), we identified adult inadequate responders if they had one of the following indicating incomplete response to initial AD: psychiatric hospitalization or emergency department (ED) visit, initiating psychotherapy, or switching to or adding on a different AD. Two mutually exclusive cohorts were identified on the basis of time from first qualifying event date to first date of augmentation with an AP (index date): 0-6 months (early add-on) and 7-12 months (late add-on). Patients were further required to be continuously enrolled 1 year before (baseline) and 1 year after (follow-up) index date. Patients with schizophrenia or bipolar disorder diagnoses were excluded. General linear regression was used to estimate adjusted healthcare costs in the early versus late add-on cohort, controlling for baseline demographic and clinical characteristics, insurance type, medications, and ED visits or hospitalizations.

Results: Of the 6935 identified inadequate responders, $68.7 \%$ started an AP early and $31.3 \%$ late. At baseline, before AP augmentation, patients in the early add-on cohort had higher psychiatric comorbid disease burden (47.3\% vs. $42.5 \% ; p<0.001)$ and higher inpatient utilization [mean (SD) $0.41(0.72)$ vs. 0.27 (0.67); $p<0.001]$ than in late add-on cohort. During follow-up, the adjusted total all-cause healthcare cost was significantly lower in the early vs. late add-on cohort $(\$ 18,864$ vs. $\$ 20,452 ; p=0.046)$. 
Conclusion: Findings of this real-world study suggest that, in patients with MDD who inadequately responded to first-line $\mathrm{AD}$ treatment, adding an AP earlier reduces overall healthcare costs.

Funding: Otsuka Pharmaceutical Development and Commercialization, Inc. and Lundbeck.

Keywords: Antipsychotics; Augmentation therapy; Healthcare costs; Major depressive disorder; Neurology

\section{INTRODUCTION}

Major depressive disorder (MDD) has a lifetime prevalence of $16 \%$ among adults in the USA and an estimated annual cost of $\$ 83$ billion $[1,2]$. Adequate treatment of MDD remains a significant challenge. Results from the Sequenced Treatment Alternatives to Relieve Depression $\left(\mathrm{STAR}^{*} \mathrm{D}\right)$ study indicated that the probability of depression remission decreases significantly after the failure of two treatment trials. About $60 \%$ of patients who continue for a second course of treatment do not respond, and after four trials, about $30 \%$ of patients remain depressed [3].

Incomplete remission of depression is associated with a higher risk of relapse, impaired work and social functioning, and an increased risk of suicide [4]. The World Health Organization rated unipolar depressive disorders as the third leading cause of disability-adjusted life years, which disproportionately accrues to individuals who have either not responded or only partially responded to first-line antidepressant (AD) treatment [2].

With patients suffering from lack of adequate response accounting for a large share of the MDD burden, developing safe and effective treatments is of great importance. Two main treatment strategies have been recommended for patients who are inadequately responding to first-line $\mathrm{AD}$. These include switching to a different $\mathrm{AD}$, favored for nonresponse [5], or augmentation with an atypical antipsychotic (AP) medication, lithium, or thyroid hormone, preferred in cases of partial response [6]. The American Psychiatric Association guidelines [7] designate the same level of confidence for augmentation with atypical APs, lithium, thyroid hormone, or another AD; however, among these agents, atypical APs have been studied in the largest number of randomized controlled trials using well-defined samples of patients with MDD [8]. In fact, the recently published Veterans Affairs Augmentation and Switching Treatments for Improving Depression Outcomes (VAST-D) trial compared augmentation with an atypical AP to an AD switch and found a slight benefit to augmentation over AD switch [9]. Prior studies focusing on inadequate treatment efficacy in MDD included patients of varying disease severity; some patients have experienced inadequate response to one $\mathrm{AD}$, while others have not responded to up to four ADs [10].

The aim of this exploratory analysis was to understand the association between timing (early vs. late) of augmentation of AD with APs and overall healthcare cost in patients with MDD following an event indicative of inadequate depression treatment efficacy (inadequate responders). In this study, we characterized events occurring prior to the addition of the adjunctive AP medication. These events were considered proxies for inadequate treatment efficacy and included AD switches or add-ons, initiation of psychotherapy, or psychiatric hospitalization or emergency department (ED) visit.

\section{METHODS}

\section{Data Source and Study Design}

We conducted a retrospective cohort study using the Truven Health MarketScan ${ }^{\circledR}$ Medicaid, Commercial, and Medicare Supplemental claims databases to compare healthcare costs in patients who initiated an oral AP presumably because of inadequate response to first-line ADs. The MarketScan Medicaid Database includes demographic and clinical information, inpatient and outpatient utilization data, and outpatient prescription data for 40 million Medicaid enrollees from multiple geographically dispersed states. To ensure complete medical claims histories, patients with Medicare dual-eligibility, capitated 
health insurance, or without mental health coverage were excluded. The MarketScan Commercial Database includes medical and pharmacy claims for approximately 65 million individuals and their dependents who are covered through employer-sponsored private health insurance plans. The MarketScan Medicare Supplemental Database contains records on about 5.3 million retired employees and spouses older than 65 years who are enrolled in Medicare with supplemental Medigap insurance paid by their former employers.

The study used medical, pharmacy, and enrollment claims from 7/1/09 through 12/31/ 16 for Medicaid data and 7/1/09 through 9/30/ 16 for Commercial and Medicare Supplemental data. All data were compliant with the Health Insurance Portability and Accountability Act (HIPAA) of 1996. Institutional review board approval was not required as MarketScan data are recorded in such a manner that subjects cannot be identified, directly or through identifiers linked to the subjects. Meeting these conditions makes this research exempt from the requirements of 45 CFR 46 under the Department of Health and Human Services (HHS): "Research, involving the collection or study of existing data, documents, records, pathological specimens, if these sources are publicly available or if the information is recorded by the investigator in such a manner that subjects cannot be identified, directly or through identifiers linked to the subjects [11]." This article does not contain any studies with human participants or animals performed by any of the authors.

\section{Sample Selection}

Patients with a diagnosis of MDD were identified if they had at least one inpatient or two outpatient medical claims for MDD (International Classification of Disease-Clinical Modification [ICD-9-CM] code: 296.2x, 296.3x; ICD-10-CM: F32.0-F32.5, F32.9, F33.0x-F33.4x, F33.9x; see Appendix Table 1 for full list of codes with descriptions) in any diagnosis field of a claim between 7/1/09 and 12/31/16 (Medicaid) or 7/1/ 09 through 9/30/16 (Commercial and Medicare Supplemental). Patients must have had evidence of inadequate treatment efficacy during the clinical event identification period (10/1/09-12/ 31/14 for Medicaid data, and 10/1/09-9/30/14 for Commercial and Medicare Supplemental data). These inadequate treatment efficacy events ("clinical events") included psychiatric hospitalization, psychiatric ED visits, initiation of psychotherapy, and AD class-level switches or augmentation (see Appendices Tables 2 and 3 for full lists of codes with descriptions). Patients with qualifying clinical events were required to have at least one $\mathrm{AD}$ medication claim during the 90 days prior to the first of the clinical events to ensure that this was a medically treated population. Only the first qualifying event for each patient was captured.

The index date, or the first date of augmentation with an oral AP, was captured up to 12 months from the clinical event. Patients were grouped into the following two cohorts based on time from first qualifying clinical event date to first date of augmentation with an AP: 0-6 months (early add-on) and 7-12 months (late add-on). All patients were required to have at least 60 days of the AP medication use within the 6 months following the index date. Additionally, to ensure that the AP was being utilized as adjunctive treatment, patients were required to have at least one AD pharmacy claim each in the 90 days prior and the 90 days after the index date, with at least 15 days overlap of AD supply with the first index oral AP prescription. Patients using combination AP therapy or those who had utilized AP medication prior to the index date were not included in the study sample. The baseline and follow-up periods were defined as the 12 months before and after the index date, respectively (Fig. 1).

Further, eligible patients were at least 18 years of age on the index date, had their first diagnosis of MDD on or before the index date, and fulfilled the requirement of 12 months of continuous enrollment both during the baseline and follow-up periods. Patients were excluded if they had at least one diagnosis of schizophrenia (ICD-9-CM codes: 295.xx, excluding $295.4 \mathrm{x}$ and $295.7 \mathrm{x}$; or ICD-10-CM codes: F20x, excluding F20.81) or bipolar I disorder (ICD-9-CM codes: 296.0x, 296.1x, 296.4x296.8x, excluding 296.82; or ICD-10-CM codes: 


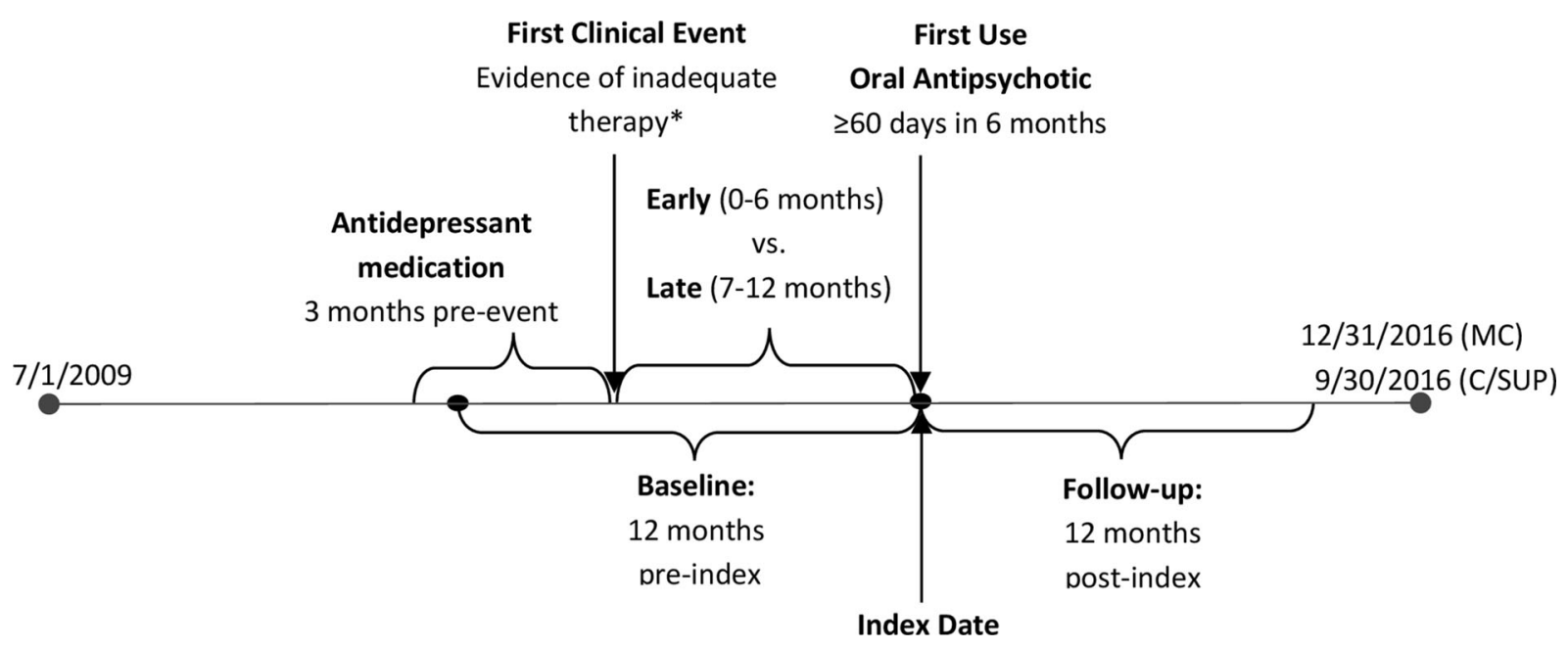

Fig. 1 Study timeline for patients with MDD who initiated adjunctive antipsychotic early vs late. The index date, or the first date of augmentation with an oral AP, was captured up to 12 months from the clinical event. All patients were required to have at least 60 days of the AP medication use within the 6 months following the index date. Additionally, to ensure that the AP was being utilized as adjunctive treatment, patients were required to have at least one $\mathrm{AD}$ pharmacy claim each in the 90 days prior and

F30.x-F31.x, excluding F31.81) anytime during the study period to account for the potentially different resource utilization and treatment patterns of these patients, compared to patients with MDD only.

\section{Study Measures}

Baseline variables, which employed data during the 12 months prior to the index date, included patient demographics (age, gender, and insurance type), event type, Charlson Comorbidity Index (CCI) $[12,13]$, number of Healthcare Cost and Utilization Project (HCUP) chronic condition indicators [14], psychiatric comorbidities (anxiety, personality disorder, and substance abuse disorder), psychiatric (antidepressants, anti-anxiety medications, sedatives or hypnotics, and mood stabilizers) and non-psychiatric (antidiabetic, lipid-lowering, and antihypertensive medications) medication use, ED visits, and hospitalizations. Unlike our patient identification algorithm (which required one inpatient or two outpatient claims the 90 days after the index date, with at least 15 days overlap of $\mathrm{AD}$ supply with the first index oral $\mathrm{AP}$ prescription. The baseline and follow-up periods were defined as the 12 months before and after the index date, respectively. ${ }^{*}$ Includes ED visits, hospital stays, initiation of psychotherapy, and/or antidepressant switches. C commercial, MC medicaid, mo months, SUP Medicare Supplemental

for MDD), when we identified patients as having psychiatric comorbidities (anxiety, personality disorder, and substance abuse disorder), the presence of a single code during the baseline period for the relevant condition was considered adequate.

The main outcome of interest was all-cause total healthcare cost and its components during the 12-month follow-up period. Total cost consisted of three main components: outpatient medical cost (outpatient and ED visits), inpatient cost (acute and non-acute inpatient stays), and outpatient pharmacy cost. Additionally, we analyzed inpatient costs among patients who experienced at least one hospitalization. All outcomes were compared between the early and late add-on study cohorts.

\section{Statistical Analysis}

Descriptive statistics were performed to assess differences between cohorts across all baseline covariates, including means and standard deviations (SD) for continuous variables, and counts 
and percentages for categorical variables. Chisquare tests and $t$ tests were utilized as appropriate. General linear regression was used to estimate the all-cause total cost during the 12-month follow-up period. Baseline covariates included age, gender, insurance type, event type, CCI, number of chronic conditions, psychiatric comorbidities (anxiety, personality disorder, substance abuse disorder), baseline psychiatric medication use (serotonin and norepinephrine reuptake inhibitors [SNRIs], selective serotonin reuptake inhibitors [SSRIs], tricyclic or tetracyclic agents, antianxiety medications, sedatives or hypnotics, mood stabilizers), hospitalizations, ED visits, and index AP class (atypical, typical). Beta coefficients, $p$ values, and 95\% confidence intervals (95\% CI) for model covariates were provided. All costs were adjusted to 2016 US dollars using the medical care component of the Consumer Price Index. All data transformations and statistical analyses were performed using SAS $\odot$ version 9.4 (Cary, $\mathrm{NC})$.

\section{RESULTS}

\section{Sample Description}

Of the $1,868,031$ patients with MDD without schizophrenia or bipolar disorder as comorbidities identified from the combined dataset (Medicaid, Commercial, and Medicare Supplemental), 515,478 experienced a clinical event indicating inadequate treatment efficacy while taking an $\mathrm{AD}$. There were 86,610 patients that had evidence of AP use within 12 months after the clinical event date. Of those patients, 30,884 had no AP use prior to the index date and filled at least 60 days of the index AP within 6 months of the index date. In total, 6935 patients met all inclusion requirements.

The most commonly prescribed index APs in this population were aripiprazole (53.4\%), quetiapine $(28.0 \%)$, risperidone $(9.5 \%)$, olanzapine (4.8\%), and ziprasidone (1.3\%). All other AP medications collectively comprised less than $5 \%$ of index AP. The early add-on cohort included $4762(68.7 \%)$ patients and the late add-on cohort consisted of 2173 (31.3\%) patients.

\section{Baseline Characteristics}

The mean age of the overall sample was 49.5 years; $68.3 \%$ of patients were female, $74.7 \%$ carried Commercial insurance, and $45.8 \%$ suffered from at least one psychiatric comorbidity (other than schizophrenia or bipolar disorder, as they were excluded), with anxiety $(40.3 \%)$ being the most common. Baseline hospitalizations occurred in $28.3 \%$ of patients, and $23.5 \%$ had a baseline ED visit. The most prevalent clinical event was initiation of psychotherapy (42.7\%). Patients in the early and late add-on cohorts differed significantly in gender, insurance type, clinical event, psychiatric comorbidities and medication use, and baseline utilization. The early add-on cohort comprised a lower percentage of women, Medicaid patients, patients who experienced an $\mathrm{AD}$ switch or add-on, patients prescribed SNRIs, other AD, mood stabilizers, and non-psychiatric medications; and a greater percentage of patients prescribed SSRIs and sedatives and hypnotics, patients with anxiety and substance abuse, and patients with ED visits and hospitalizations $(p<0.05$ for all comparisons $)$ (Table 1).

\section{Timing of Antipsychotic Initiation and All-Cause Cost: Univariate and Multivariate Analysis}

The mean unadjusted all-cause cost during the 12-month follow-up period was not statistically significantly different when comparing the early and late add-on cohorts [mean (SD): $\$ 18,842 \quad(27,886) \quad$ vs. $\$ 20,500 \quad(38,127)$ $p=0.069]$. While all components of the total cost were numerically lower in the early add-on cohort, the difference was only statistically significant for outpatient pharmacy costs [mean (SD): $\$ 6328$ (6185) vs. \$7091 (6305), $p<0.001]$. The cost of hospitalization among those who were hospitalized in the early add-on cohort was $\$ 24,138(39,296)$ vs. $\$ 30,516(67,037)$ in the late add-on cohort ( $p=0.054)$ (Table 2). 
Table 1 Baseline demographics, clinical characteristics, and utilization of early vs. late add-on cohorts

\begin{tabular}{|c|c|c|c|c|}
\hline & $\begin{array}{l}\text { Early add-on (within } \\
6 \text { months) }\end{array}$ & $\begin{array}{l}\text { Late add- } \\
\text { on }\end{array}$ & All & $p$ value \\
\hline$N$ & 4762 & 2173 & 6935 & \\
\hline$\%$ & 68.7 & 31.3 & 100.0 & \\
\hline \multicolumn{5}{|l|}{ Age, year } \\
\hline Mean & 49.4 & 49.6 & 49.5 & 0.612 \\
\hline (SD) & $(15.8)$ & $(15.1)$ & $(15.6)$ & \\
\hline [Median] & {$[50]$} & {$[50]$} & {$[50]$} & \\
\hline \multicolumn{5}{|l|}{ Female } \\
\hline No. & 3170 & 1564 & 4734 & $<0.001$ \\
\hline (\%) & $(66.6)$ & $(72.0)$ & $(68.3)$ & \\
\hline \multicolumn{5}{|c|}{ Insurance type } \\
\hline \multicolumn{5}{|l|}{ Medicaid } \\
\hline No. & 498 & 280 & 778 & 0.008 \\
\hline (\%) & $(10.5)$ & $(12.9)$ & $(11.2)$ & \\
\hline \multicolumn{5}{|c|}{ Commercial } \\
\hline No. & 3574 & 1603 & 5177 & \\
\hline (\%) & $(75.1)$ & $(73.8)$ & $(74.7)$ & \\
\hline \multicolumn{5}{|c|}{ Medicare Supplemental } \\
\hline No. & 690 & 290 & 980 & \\
\hline (\%) & $(14.5)$ & $(13.3)$ & $(14.1)$ & \\
\hline \multicolumn{5}{|c|}{ Pre-index event } \\
\hline \multicolumn{5}{|c|}{ Psychiatric-related inpatient hospitalization } \\
\hline No. & 709 & 113 & 822 & $<0.001$ \\
\hline$(\%)$ & $(14.9)$ & $(5.2)$ & $(11.9)$ & \\
\hline \multicolumn{5}{|c|}{ Psychiatric-related ED visit } \\
\hline No. & 222 & 83 & 305 & \\
\hline$(\%)$ & $(4.7)$ & $(3.8)$ & $(4.4)$ & \\
\hline \multicolumn{5}{|c|}{ Psychotherapy } \\
\hline No. & 2061 & 897 & 2958 & \\
\hline$(\%)$ & $(43.3)$ & $(41.3)$ & $(42.7)$ & \\
\hline \multicolumn{5}{|c|}{ Switch/add-on antidepressant } \\
\hline No. & 1770 & 1080 & 2850 & \\
\hline$(\%)$ & $(37.2)$ & $(49.7)$ & $(41.1)$ & \\
\hline
\end{tabular}


Table 1 continued

Early add-on (within

6 months)

Index oral antipsychotic

Atypical antipsychotic

No.

(\%)

4681

(98.3)

81

(1.7)

(1.7)

(\%)

Charlson Comorbidity Index (CCI)

Mean

0.8

(SD)

No. chronic conditions (HCUP)

Mean

3.2

(SD)

Psychiatric comorbidities

No.

2252

(\%)

(47.3)

Anxiety

No.

(\%)

Personality disorder

No.

(\%)

Substance abuse disorders

No.

(\%)
554

(11.6) on

2135

(98.3)

0.7

(1.5)

3.3

(2.0)

924

(42.5)

1976

(41.5)

820

(37.7)

104

43

(2.0)

212

Late add- All $p$ value

6816

0.887

(98.3)

119

(1.7)

0.8

0.510

(1.4)

$3.3 \quad 0.488$

(1.9)

$3176<0.001$

(45.8)

$2796 \quad 0.003$

(40.3)

147

0.582

$(2.1)$

766

0.021

Non-psychiatric comorbidities (includes obesity, diabetes mellitus, type 2, hyperlipidemia, and hypertension)

No.

(\%)

No. of baseline ED visits
Mean
0.41
(SD)

1014

3265

(47.1)

(46.7)

0.40

0.41

$0.014^{\mathrm{a}}$

(1.27)

(1.10) 
Table 1 continued

\begin{tabular}{|c|c|c|c|c|}
\hline & $\begin{array}{l}\text { Early add-on (within } \\
6 \text { months) }\end{array}$ & $\begin{array}{l}\text { Late add- } \\
\text { on }\end{array}$ & All & $p$ value \\
\hline \multicolumn{5}{|l|}{$\geq 1$} \\
\hline No. & 1162 & 367 & 1629 & 0.085 \\
\hline$(\%)$ & $(24.4)$ & $(21.5)$ & $(23.5)$ & \\
\hline \multicolumn{5}{|c|}{ No. of baseline inpatient hospitalizations } \\
\hline Mean & 0.41 & 0.27 & 0.37 & $<0.001^{\mathrm{a}}$ \\
\hline$(\mathrm{SD})$ & $(0.72)$ & $(0.67)$ & $(0.71)$ & \\
\hline \multicolumn{5}{|l|}{$\geq 1$} \\
\hline No. & 1546 & 420 & 1966 & $<0.001$ \\
\hline$(\%)$ & $(32.5)$ & $(19.3)$ & $(28.3)$ & \\
\hline
\end{tabular}

Antidepressants

Monoamine oxidase inhibitors

No.

(\%)

Serotonin-norepinephrine reuptake inhibitors (SNRIs)

No.

(\%)

Selective serotonin reuptake inhibitors (SSRIS)

No.

(\%)

3301

(69.3)

Tricyclic or tetracyclic agents

No.

(\%)

Combination antidepressants

No.

(\%)

Other antidepressants

No.

$$
\text { (\%) }
$$

1940

(40.7)

$(0.0)$

9

(0.1)

$(0.2)$

$(69.3)$

876

$(18.4)$

\section{9}

(1)

Anti-anxiety medications

No.

(\%)
2332

(49.0)

\section{6}

(45.4)

1327

$4628<0.001$

(61.1)

(66.7)

$2826<0.001$

(40.7)

415

1291

0.486

(19.1)

(18.6)

6

15

0.469

(0.3)

(0.2)

1044

$2984<0.001$

(48.0)

(43.0)

1041

3373

0.411

(47.9) 
Table 1 continued

$\begin{array}{ll}\text { Early add-on (within } & \text { Late add- All } \\ 6 \text { months) } & \text { on }\end{array}$

Sedatives or hypnotics

No. 1534

(\%)

Mood stabilizers

No.

404

(\%)
646

(29.7)

247

$(11.4)$

$2180 \quad 0.039$

(31.4)

$651<0.001$

Non-psychiatric medications (includes antidiabetic, lipid-lowering, and antihypertensive medications)

\begin{tabular}{|c|c|c|c|}
\hline No. & 2512 & 1202 & 3714 \\
\hline$(\%)$ & $(52.8)$ & $(55.3)$ & $(53.6)$ \\
\hline
\end{tabular}

ED emergency department, HCUP Healthcare Cost and Utilization Project, SD standard deviation

After adjusting for differences in baseline characteristics, the timing of adjunctive AP initiation (early vs. late cohort) was a significant predictor of all-cause cost. The early add-on cohort incurred $\$ 1587$ less in total during the 12-month follow-up period (95\% CI - 3148 to - 26) than the late add-on cohort. The adjusted total all-cause cost was $\$ 18,864$ for the early add-on cohort and $\$ 20,452$ for the late add-on cohort (Table 3).

\section{DISCUSSION}

We performed an exploratory analysis to investigate cost outcomes in patients who initiated an oral AP medication early versus late following an event suggestive of inadequate depression treatment efficacy. The early add-on cohort seemed to be sicker and have a greater burden of psychiatric disease. Considering the triggering events denoting treatment inadequacy, higher percentages of patients in the early add-on cohort experienced a psychiatric admission or ED visit, as well as initiation of psychotherapy; a lower percentage of patients in the early add-on cohort switched or added on an $\mathrm{AD}$, when compared with the late add-on cohort. Further, on univariate analysis of baseline characteristics, the early add-on cohort also showed signs of more complicated psychiatric disease, reflected as higher rates of psychiatric comorbidities; however, this result is tempered by the incorporation of psychiatric exclusion criteria. In multivariate analysis, the patients who initiated an oral AP medication early (within 6 months) demonstrated lower all-cause total healthcare costs during the ensuing 12 months, compared to those who were prescribed an oral AP later [mean (SD) $\$ 18,864$ $(18,004-19,725)$ vs. $\$ 20,452(19,167-21,736)$, $p=0.046]$.

The early add-on cohort in this retrospective study was associated with lower total all-cause cost during the first year of AP use, suggesting that earlier augmentation could decrease costs. If further studies were to support these findings, it would suggest that earlier augmentation with AP medication could be superior to AD switch in patients with inadequate response to ADs. This finding is consistent with the emerging evidence of benefit to augmentation over AD switch [9]. Patients in the early add-on cohort had lower percentages of AD switches and addons prior to initiation of AP medication, as compared with the late add-on cohort $(37.2 \%$ vs. $49.7 \%, p<0.001)$. Although all patients in this study were prescribed oral AP augmentation, it is possible the early add-on cohort consisted of patients who were more rapidly 
Table 2 Unadjusted all-cause cost for early vs late add-on cohorts during the 12-month follow-up period

\begin{tabular}{|c|c|c|c|c|}
\hline & $\begin{array}{l}\text { Early add-on } \\
\text { (within } 6 \text { months) }\end{array}$ & Late add-on & All & $p$ value \\
\hline$N$ & 4762 & 2173 & 6935 & \\
\hline \multicolumn{5}{|c|}{ All-cause total cost } \\
\hline Mean & $\$ 18,842$ & $\$ 20,500$ & $\$ 19,362$ & 0.069 \\
\hline (SD) & $(27,886)$ & $(38,127)$ & $(31,462)$ & \\
\hline [Median] & {$[11,263]$} & {$[11,983]$} & {$[11,522]$} & \\
\hline \multicolumn{5}{|c|}{ Outpatient medical cost } \\
\hline Mean & $\$ 8357$ & $\$ 8623$ & $\$ 8440$ & 0.600 \\
\hline (SD) & $(15,507)$ & $(21,177)$ & $(17,482)$ & \\
\hline [Median] & {$[4281]$} & {$[4268]$} & {$[4272]$} & \\
\hline \multicolumn{5}{|c|}{ Inpatient cost } \\
\hline Mean & $\$ 4248$ & $\$ 4786$ & $\$ 4416$ & 0.414 \\
\hline$(\mathrm{SD})$ & $(18,742)$ & $(27,972)$ & $(22,053)$ & \\
\hline$[$ Median $]$ & {$[0]$} & {$[0]$} & {$[0]$} & \\
\hline \multicolumn{5}{|c|}{ Inpatient hospital costs among patients with $\geq 1$ inpatient hospitalization } \\
\hline$N$ & 758 & 311 & 1069 & \\
\hline Mean & $\$ 24,138$ & $\$ 30,516$ & $\$ 25,994$ & 0.054 \\
\hline (SD) & $(39,296)$ & $(67,037)$ & $(49,065)$ & \\
\hline [Median] & {$[11,647]$} & {$[14,377]$} & {$[12,717]$} & \\
\hline \multicolumn{5}{|c|}{ Outpatient pharmacy costs } \\
\hline Mean & $\$ 6238$ & $\$ 7091$ & $\$ 6505$ & $<0.001$ \\
\hline (SD) & $(6185)$ & $(6305)$ & $(6235)$ & \\
\hline [Median] & {$[4872]$} & {$[5835]$} & {$[5182]$} & \\
\hline
\end{tabular}

$S D$ standard deviation

augmented with oral APs and may have experienced fewer trials of $\mathrm{AD}$ switches. Instead of focusing on the number of failed AD trials, we characterized the absolute timing from an event indicating suboptimal response to current therapy, and the associated economic effects, adding these important data to the literature.

Our observations suggest that physicians are adhering to the evidence and augmenting more complicated or less treatment-responsive patients earlier than those who are less severe. The early add-on cohort had more complex psychiatric disease and tended toward ED visits and inpatient admissions; patients in the early add-on cohort experienced a psychiatric-related hospitalization prior to AP initiation at nearly three times the rate of the late add-on cohort $(14.9 \%$ vs. $5.2 \%, p<0.001)$. The faster onset and improved effects of adjunctive APs may be related to why these more severe patients initiated these medications earlier. AP medications are known for their relatively rapid onset, showing clinical benefits within 1-2 weeks [15], and these effects are at doses as low as a quarter 
Table 3 Adjusted all-cause costs for early vs. late add-on cohorts during the 12-month follow-up period

\begin{tabular}{|c|c|c|}
\hline & \multicolumn{2}{|c|}{$\begin{array}{l}\text { Total all-cause healthcare costs during } \\
\text { 12-month follow-up }\end{array}$} \\
\hline & Estimate $(95 \% \mathrm{CI})$ & $p$ value \\
\hline \multicolumn{3}{|l|}{ Age } \\
\hline $18-34$ vs $55+$ & $\$-617(-3021$ to 1786$)$ & 0.615 \\
\hline $35-44$ vs $55+$ & $\$-1516(-3758$ to 727$)$ & 0.185 \\
\hline $45-54$ vs $55+$ & $\$-224(-2251$ to 1802$)$ & 0.828 \\
\hline Female vs male & $\$ 403(-1140$ to 1945$)$ & 0.609 \\
\hline \multicolumn{3}{|l|}{ Insurance type } \\
\hline $\mathrm{MCD}$ vs $\mathrm{COM}$ & $\$-1714(-4119$ to 691$)$ & 0.162 \\
\hline $\mathrm{MCR}$ vs $\mathrm{COM}$ & $\$-1447(-3955$ to 1061$)$ & 0.258 \\
\hline \multicolumn{3}{|l|}{ Pre-index event } \\
\hline Psychiatric-related inpatient hospitalization vs switch/add-on antidepressant & $\$-2524(-5269$ to 222$)$ & 0.072 \\
\hline Psychiatric-related ED visit vs switch/add-on antidepressant & $\$ 439(-3349$ to 4227$)$ & 0.820 \\
\hline Psychotherapy vs switch/add-on antidepressant & $\$ 335(-1298$ to 1969$)$ & 0.687 \\
\hline Atypical antipsychotic vs typical antipsychotic & $\$-808(-6284$ to 4669$)$ & 0.772 \\
\hline Charlson comorbidity index (CCI) & $\$ 3464(2854$ to 4074$)$ & $<0.001$ \\
\hline No. chronic conditions (HCUP) & $\$ 2388$ (1896 to 2880$)$ & $<0.001$ \\
\hline Anxiety (yes vs no) & $\$-1151(-2678$ to 376$)$ & 0.140 \\
\hline Personality disorder (yes vs no) & $\$ 306(-4712$ to 5325$)$ & 0.905 \\
\hline Substance abuse disorder (yes vs no) & $\$ 1932(-500$ to 4364$)$ & 0.119 \\
\hline Serotonin-norepinephrine reuptake inhibitors (yes vs no) & $\$ 1068(-637$ to 2774$)$ & 0.220 \\
\hline Selective serotonin reuptake inhibitors (yes vs no) & $\$-692(-2454$ to 1070$)$ & 0.441 \\
\hline Tricyclic or tetracyclic agents (yes vs no) & $\$ 2464(567$ to 4361$)$ & 0.011 \\
\hline Antianxiety medications (yes vs no) & $\$ 1213(-263$ to 2690$)$ & 0.107 \\
\hline Sedatives or hypnotics (yes vs no) & $\$ 1808$ (247 to 3368$)$ & 0.023 \\
\hline Mood stabilizers (yes vs no) & $\$ 4793$ (2334 to 7252$)$ & $<0.001$ \\
\hline Any baseline inpatient hospitalization (yes vs no) & $\$ 2561(513$ to 4610$)$ & 0.014 \\
\hline Any baseline ED visit (yes vs no) & $\$ 1605(-236$ to 3445$)$ & 0.087 \\
\hline Early vs late add-on & $\$-1587(-3148$ to -26$)$ & 0.046 \\
\hline
\end{tabular}


Table 3 continued

\begin{tabular}{llc}
\hline & \multicolumn{2}{l}{ Total all-cause healthcare costs during 12-month follow-up } \\
\cline { 2 - 3 } & Adjusted mean (95\% CI) ${ }^{\mathbf{a}}$ & $\boldsymbol{p}$ value \\
\hline Comparison group & & 0.046 \\
Early add-on (within 6 months) & $\$ 18,864(18,004-19,725)$ & \\
Late add-on & $\$ 20,452(19,167-21,736)$ & \\
\hline
\end{tabular}

95\% CI 95\% confidence interval, COM commercial insurance, ED emergency department, HCUP Healthcare Cost and Utilization Project, $M C D$ Medicaid, $M C R$ Medicare

to half of those used to treat acute schizophrenia or mania [16]. A recent study found that augmentation with an atypical AP medication demonstrated added benefit in patients with a higher degree of treatment-resistant depression (TRD) [17]. Further, a study by McIntyre et al., based on physician surveys, found that one of the main predictive factors that led to the prescription of an AP medication in MDD was severity of illness [18]. Using insurance claims data, our findings demonstrate higher rates of patients with more severe disease undergoing earlier AP augmentation, validating the results of the previous survey-based study.

The adjusted all-cause total costs for the 1-year period following oral AP initiation were $\$ 18,864$ for the early add-on cohort and $\$ 20,452$ for the late add-on cohort; these values provide an updated cost of MDD treated with oral APs. A previous study utilizing data from the Medicare population with managed depression found the yearly total cost to be $\$ 13,252$ [19]. Since this cohort comprised patients with managed depression, it is likely that less severe, and therefore, less expensive patients are included. Halpern et al. investigated total medical costs of various adjunctive APs in a commercial population; the 1-year costs ranged from $\$ 10,664$ to $\$ 14,583$ [20]. Our costs were higher than these studies, which could stem from the dataset utilized. Our study population combined patients with Medicaid, Medicare, and Commercial insurance. The study by Halpern et al. better matches our methodology, in that costs are measured from the initiation of the AP medication; however, this study was performed using a population with Commercial insurance, likely causing the costs to be lower. Additionally, the previous analyses were performed several years prior and healthcare costs have continued to increase over time.

Several limitations of this study stem from the health insurance claims dataset utilized. First, we were unable to directly measure disease severity; we were not only unable to ascertain the level of a patient's MDD but also could not determine when a patient entered remission. We did, however, use a variety of measures to adjust for severity, including baseline non-psychiatric and psychiatric comorbidities, pre-index event, medication use, and inpatient and ED utilization. There may have been additional differences we could not measure or adjust for in models. Second, because we were unable to assess MDD severity directly, we identified proxies indicative of inadequate treatment efficacy, such as psychiatric hospitalizations or ED visits. Third, increased rates of side effects, when compared to ADs, are an important aspect of AP use to identify when investigating the outcomes surrounding augmentation and switching. Insurance claims data are not an ideal source to capture patients who discontinued AP medications secondary to side effects or intolerability. In fact, given the requirement for at least 60 days of AP use during the 6 months following the index date, many patients who may have suffered from intolerable side effects were likely excluded from the analysis. This requirement was employed as a way to fairly adjust for the severity of the study population; a 
patient who utilized only a week of AP medication likely had different experiences and costs than a patient who adhered for several months. Lastly, all MDD diagnoses were identified through health insurance claims data, where misclassification, diagnostic uncertainty, or coding errors are possible. Although claims data do have the aforementioned limitations, studies utilizing these data include more diverse individuals who may better characterize conditions and their treatment in a real-world setting, as opposed to the relatively homogenous populations that tend to comprise clinical trials. Despite these limitations, we believe that this initial exploratory analysis provides valuable insight into cost outcomes associated with the timing of oral AP augmentation.

\section{CONCLUSIONS}

This study investigated the differences between patients with MDD who initiated oral AP augmentation early versus late following an event indicative of inadequate depression treatment efficacy. Patients in the early add-on cohort, despite their higher levels of both baseline psychiatric comorbidities and hospitalizations, had significantly lower costs during the 1-year period following oral AP medication initiation in a real-world study of a population that combined patients with Medicaid, Medicare, and Commercial insurance. Further studies are warranted to more thoroughly characterize this association between earlier oral AP medication initiation and lower all-cause total cost.

\section{ACKNOWLEDGEMENTS}

Funding. Funding for the study and article processing fees were received from Otsuka Pharmaceutical Development and Commercialization, Inc. and Lundbeck. All authors had full access to all of the data in this study and take complete responsibility for the integrity of the data and accuracy of the data analysis.
Authorship. All authors met the ICMJE criteria for authorship, including contributing to study concept and design, interpretation of the data, and to the drafting and critical review of the manuscript. E. Chang further contributed to the analysis of the data. This manuscript has been read and approved by all authors.

Disclosures. Michael S. Broder is an employee of PHAR, LLC, which was paid by Otsuka and Lundbeck to perform the research described in this manuscript. Tingjian Yan is an employee of PHAR, LLC, which was paid by Otsuka and Lundbeck to perform the research described in this manuscript. Eunice Chang is an employee of PHAR, LLC, which was paid by Otsuka and Lundbeck to perform the research described in this manuscript. Irina Yermilov is an employee of PHAR, LLC, which was paid by Otsuka and Lundbeck to perform the research described in this manuscript. Mallik Greene is an employee of Otsuka Pharmaceutical Development and Commercialization, Inc. Ann Hartry is an employee of Lundbeck.

Compliance with Ethics Guidelines. This article does not contain any studies with human participants or animals performed by any of the authors.

Data Availability. The datasets generated during and/or analyzed during the current study are not publicly available as the Truven Health data were used under license for the current study, but are available from the authors upon reasonable request and with permission of Truven Health.

Open Access. This article is distributed under the terms of the Creative Commons Attribution-NonCommercial 4.0 International License (http://creativecommons.org/licenses/ by-nc/4.0/), which permits any noncommercial use, distribution, and reproduction in any medium, provided you give appropriate credit to the original author(s) and the source, provide a link to the Creative Commons license, and indicate if changes were made. 


\section{REFERENCES}

1. Kennedy SH. A review of antidepressant therapy in primary care: current practices and future directions. Prim Care Companion CNS Disord. 2013;15(2). https://doi.org/10.4088/PCC.12r01420.

2. McIntyre RS, Filteau M-J, Martin L, et al. Treatmentresistant depression: definitions, review of the evidence, and algorithmic approach. J Affect Disord. 2014;156:1-7.

3. Rush A, Trivedi M, Wisniewski S, et al. Acute and longer-term outcomes in depressed outpatients requiring one or several treatment steps: a STAR ${ }^{\star} \mathrm{D}$ report. Am J Psychiatry. 2006;163:1905-17.

4. Thase ME. Treatment-resistant depression: prevalence, risk factors, and treatment strategies. J Clin Psychiatry. 2011;72:e18.

5. Wisniewski SR, Fava M, Trivedi MH, et al. Acceptability of second-step treatments to depressed outpatients: a STAR ${ }^{\star} \mathrm{D}$ report. Am J Psychiatry. 2007;164:753-60.

6. Shelton RC, Osuntokun O, Heinloth AN, Corya SA. Therapeutic options for treatment-resistant depression. CNS Drugs. 2010;24:131-61.

7. American Psychiatric Association. Practice guideline for the treatment of patients with major depressive disorder. Washington, DC: American Psychiatric Publishing; 2010.

8. Patkar AA, Pae C-U. Atypical antipsychotic augmentation strategies in the context of guidelinebased care for the treatment of major depressive disorder. CNS Drugs. 2013;27:29-37.

9. Mohamed S, Johnson GR, Chen P, et al. Effect of antidepressant switching vs augmentation on remission among patients with major depressive disorder unresponsive to antidepressant treatment: the VAST-D randomized clinical trial. JAMA. 2017;318:132.

10. Zhou X, Ravindran AV, Qin B, et al. Comparative efficacy, acceptability, and tolerability of augmentation agents in treatment-resistant depression: systematic review and network meta-analysis. J Clin Psychiatry. 2015;e487-98.

11. Office for Human Research Protections. Coded private information or specimens use in research, guidance (2008). HHS.gov. 2008. https://www.hhs. gov/ohrp/regulations-and-policy/guidance/ research-involving-coded-private-information/ index.html. Accessed 26 Oct 2018.

12. Charlson ME, Pompei P, Ales KL, MacKenzie CR. A new method of classifying prognostic comorbidity in longitudinal studies: development and validation. J Chron Dis. 1987;40:373-83.

13. Deyo RA, Cherkin DC, Ciol MA. Adapting a clinical comorbidity index for use with ICD-9-CM administrative databases. J Clin Epidemiol. 1992;45:613-9.

14. Agency for Healthcare Research and Quality. HCUP chronic condition indicator. Healthcare Cost and Utilization Project. HCUP. 2015. www.hcup-us. ahrq.gov/toolssoftware/chronic/chronic.jsp. Accessed 22 May 2018.

15. Thase ME. Adverse effects of second-generation antipsychotics as adjuncts to antidepressants. Psychiatr Clin North Am. 2016;39:477-86.

16. Connolly KR, Thase ME. If at first you dont succeed: a review of the evidence for antidepressant augmentation, combination and switching strategies. Drugs. 2011;71:43-64.

17. Wang HR, Woo YS, Ahn HS, Ahn IM, Kim HJ, Bahk W-M. Can atypical antipsychotic augmentation reduce subsequent treatment failure more effectively among depressed patients with a higher degree of treatment resistance? A meta-analysis of randomized controlled trials. Int $\mathrm{J}$ Neuropsychopharmacol. 2015;18(8). https://doi.org/10. 1093/ijnp/pyv023.

18. McIntyre RS, Weiller E. Real-world determinants of adjunctive antipsychotic prescribing for patients with major depressive disorder and inadequate response to antidepressants: a case review study. Adv Ther. 2015;32:429-44.

19. Feldman RL, Dunner DL, Muller JS, Stone DA. Medicare patient experience with vagus nerve stimulation for treatment-resistant depression. J Med Econ. 2013;16:62-74.

20. Halpern R, Nadkarni A, Kalsekar I, et al. Medical costs and hospitalizations among patients with depression treated with adjunctive atypical antipsychotic therapy: an analysis of health insurance claims data. Ann Pharmacother. 2013;47:933-45. 